In der Rubrik „Literatur kompakt" werden die wichtigsten Originalarbeiten aus der internationalen Fachliteratur referiert.

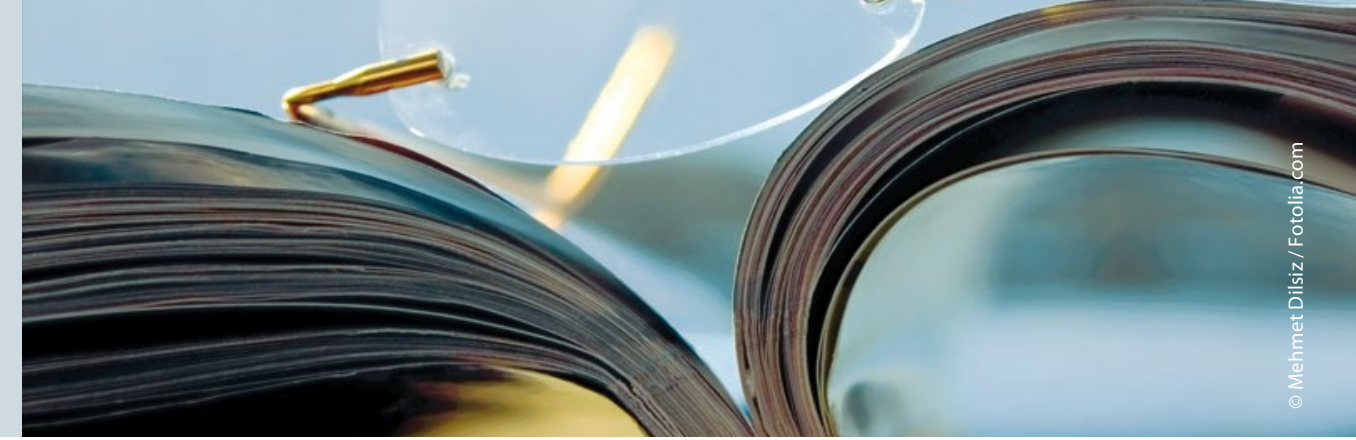

\title{
Mundhöhlenkrebs: Die Lymphknoten gleich mit entfernen?
}

\begin{abstract}
Elektive neck dissection oder "watchful waiting"? Welche Strategie bei Plattenepithelkarzinomen in der Mundhöhle im Frühstadium nach einer Exzision des Primärtumors den größeren Vorteil bietet, haben indische Forscher in einer prospektiven und randomisierten Phase-III-Studie untersucht.
\end{abstract}

5 a atienten mit einem invasiven Plattenepithelkarzinom der Mundhöhle und negativem klinischem Lymphknotenbefund (T1, T2) haben grundsätzlich zwei Optionen: Entweder werden im Zuge der Resektion des Primärtumors prophylaktisch gleich die Lymphknoten mehrerer Ebenen im Halsbereich und das umgebende Gewebe mit herausgenommen (elektive neck dissection). Oder man beschränkt sich im Sinne des „watchful waiting " zunächst auf die Entfernung des Primärtumors; die Halslymphknoten bleiben in diesem Fall erst einmal erhalten und werden erst dann entfernt, wenn sie von Metastasen befallen sind (therapeutische neck dissection).

Anil K. D'Cruz und Kollegen vom Tata Memorial Centre in Mumbai, Indien, haben beide Vorgehensweisen in einer prospektiven, randomisierten Studie mit 500 Patienten verglichen. 245 wurden der elektiven Dissektion zugeteilt, 255 der therapeutischen Variante. Zunächst wurde bei allen Patienten der Primärtumor oral exzidiert. Bei den Patienten der prophylaktischen Gruppe wurden zusätzlich ipsilateral die submandibulären, kranio-jugulären und medio-jugulären Lymphknoten (Ebene I bis III) ausgeräumt, bei intraoperativ entdecktem Lymphknotenbefall auch die Ebenen IV und V. Die therapeutische
Gruppe wurde nach der Primäroperation zunächst nur überwacht.

Nach drei Jahren hatten $80 \%$ der elektiv operierten Patienten überlebt, in der Gruppe mit therapeutischer Dissektion waren es $67,5 \%$. Damit war bei der Gruppe mit der elektiven neck dissection das Gesamtmortalitätsrisiko um $36 \%$ reduziert (Hazard Ratio [HR] 0,64; $95 \%$-Konfidenzintervall [95\%-KI] 0,45$0,92 ; \mathrm{p}=0,01) \cdot 69,5 \%$ der elektiven Gruppe waren nach drei Jahren noch rezidivfrei gegenüber nur $45,9 \%$ in der Vergleichsgruppe (HR 0,45; $95 \%$-KI $0,34-0,59 ; \mathrm{p}<0,001)$. Auch nach statistischer Kontrolle für verschiedene Einflussgrößen (Tumordifferenzierung, Resektionsrand, Invasionstiefe) blieben die Unterschiede zwischen den beiden Gruppen signifikant.

114 „watchful-waiting“-Patienten entwickelten Metastasen in den Halslymphknoten, woraufhin sie eine modifizierte neck dissection der Ebenen I bis V erhielten. Von diesen Patienten starben 52,6\% an ihrer fortschreitenden Erkrankung.

Die Mehrzahl aller Patienten hatte ein Zungenkarzinom als Primärtumor (85,3\%); die Ergebnisse gelten also insbesondere für diese Patientengruppe.

Unerwünschte Ereignisse hielten sich in beiden Gruppen in Grenzen: Die Rate der Komplikationen betrug bei den ab-

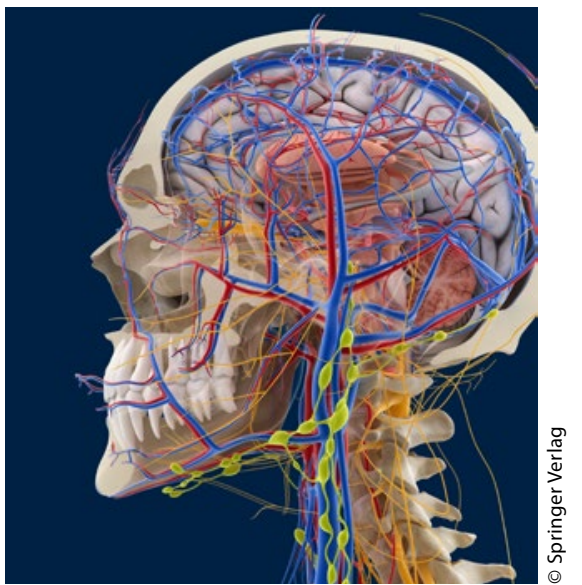

Die vorsorgliche Entfernung der Halslymphknoten (elektive neck dissection) bringt einen Überlebensvorteil für die Patienten

wartend behandelten Patienten 3,6\%, in der Gruppe mit elektiver Dissektion 6,6\%. Dazu zählten u.a. orale Blutungen (jeweils 1 Fall), postoperative Infektionen (1 bzw. 2 Fälle) sowie 3 Hämatome und 4 durch die adjuvante Radiotherapie bedingte Langzeitfolgen in der elektiven Gruppe.

Fazit: Mit der Strategie des „watchful waiting“ nach Exzision des Primärtumors kann man zwar vielen Patienten mit Mundhöhlenkarzinom einen zusätzlichen und sehr belastenden operativen Eingriff ersparen. Allerdings spricht der Überlebensvorteil in der vorliegenden Studie für eine elektive „neck dissection“ mit Ausräumung der ipsilateralen Lymphknoten bereits zum Zeitpunkt der Primäroperation.

Elke Oberhofer

$D^{\prime}$ Cruz AK et al. Elective versus therapeutic neck dissection in node-negative oral cancer. N Engl J Med. 2015;373(6):521-9. 